\title{
Investigating the role of neuropathic pain relief in decreasing gait variability in diabetes mellitus patients with neuropathic pain: a randomized, double-blind crossover trial
}

Surshen Karmakar, Houman Rashidian, Cynthia Chan, CaiXia Liu and Cory Toth*

\begin{abstract}
Background: Subjects with diabetes mellitus (DM) develop gait dysfunction contributing to falls, reluctance to perform activities and injuries. Neuropathic pain (NeP) related to diabetic peripheral neuropathy (DPN) is associated with increased gait variability that may contribute to gait dysfunction. We used a portable device (GaitMeter ${ }^{\mathrm{TM}}$ ) and related gait and balance measures to measure gait parameters in painful DPN (PDPN) subjects prior to and during analgesia. Our hypothesis was that PDPN subjects would have decreased gait step variability when receiving pharmacological relief of NeP.
\end{abstract}

Methods: DPN subjects with at least moderate NeP were assessed in a randomized, double-blind crossover study of pregabalin versus placebo. The outcome measure was variability in step length and step velocity. Testing for Timed Get-Up-and-Go Test, Tinetti Mobility Scales, Sway Testing, a Physiological Profile Approach, and fall-related surveys were also performed. DPN severity was quantified using the Utah Early Neuropathy Score.

Results: PDPN subjects developed increased, rather than decreased, step length and step velocity variability during pregabalin treatment. There were no significant differences between cohorts for other physiological gait and balance testing. Non-significant NeP relief occurred in the pregabalin phase of study as compared with placebo. There was a negative relationship for step length with pain severity.

Conclusion: Analgesia did not decrease gait variability in PDPN patients, and in fact, increased gait variability was seen during pregabalin treatment. Other important relationships of gait dysfunction with PDPN should be sought.

Keywords: Type 2 diabetes mellitus, Gait, Diabetic neuropathy, Diabetic neuropathic pain, Pregabalin

\section{Introduction}

A strong predictor for falling is the presence of diabetes mellitus (DM) [1,2]. Falling may occur in DM patients due to its many systemic complications including hypoglycaemia, orthostatic hypotension, obesity, cardiovascular disease, vestibular dysfunction, visual impairment, cognitive impairment [3], and age-related decline. However, another important contributor may be diabetic peripheral neuropathy (DPN), present in up to half of DM

\footnotetext{
*Correspondence: corytoth@shaw.ca

Department of Clinical Neurosciences, HMRB 155, Foothills Hospital,

University of Calgary, Hotchkiss Brain Institute, 3330 Hospital Dr. NW, Calgary T2N 4 N1, AB, Canada
}

causing sensory, motor and autonomic dysfunction [4] impacting upon gait function [5].

Within DM subjects, those with DPN are more likely to suffer falls [6]. Although possibly related to reduction in proprioceptive input, other factors such as neuropathic pain (NeP) related to DPN may play roles. NeP, defined as pain initiated by a lesion or dysfunction of the nervous system [7], is present in $25-50 \%$ of DPN patients [8]. NeP in DPN (PDPN) leads to an "antalgic gait" [9], assumed in order to circumvent or decrease pain while walking. Antalgic gait is associated with reduced gait velocity, shorter cadence and step length, with less rhythmic accelerations [10]. Although initially appearing to be a more stable gait, an antalgic gait may actually have greater 
gait variability and higher risks of falling [11]. Gait variability, defined as the extent to which gait parameters such as step length or step velocity diverge from a mean value, has been previously investigated by our group with reference to PDPN. We have previously hypothesized that gait dysfunction, defined as abnormal or impaired functioning of walking or running, may be associated with greater extents of gait variability [9]. Our previous work has demonstrated that variability of step length and velocity increases in PDPN subjects when compared to a cohort of painless DPN subjects and control subjects [9], even though other forms of chronic pain, such as with low back pain, can contribute to reduced variability [12]. Thus, we postulated that $\mathrm{NeP}$, a potentially treatable risk factor, is contributory to gait variability, gait dysfunction, and the subsequent risk of falling.

We hypothesized that patients with PDPN would have decreased gait variability with pharmacological NeP management using pregabalin, an indicated therapy for PDPN. If pain due to PDPN is itself responsible for the increased gait variability, then it could be theorized that reduced pain severity would reduce such variability to levels seen in DPN patients without pain. We performed a randomized, double-blinded, cross-over study comparing the effect of pregabalin, an effective pharmacotherapy for PDPN, compared with placebo intervention. Gait variability was assessed using GaitMeter ${ }^{\mathrm{rm}}$, a wireless, portable gait detection system previously studied in control subjects, DM patients, and patients with DPN [9], with reliability for its use in determining gait variability.

\section{Methods}

\section{Subject recruitment}

This study was a single-center, double-blind, randomized, placebo-controlled cross-over clinical trial. Two periods of six-week flexible-dose trials of pregabalin/ placebo were compared using the same subject as comparator, with two week washout phases intervening. Ethical approval for this study was received from the University of Calgary Centre for Advancement of Health. Participant recruitment took place from August 2011January 2013 using poster recruitment at the tertiary care clinics within the University of Calgary. Informed written consent was received from all participants prior to involvement. Each subject had pre-existing type 2 diabetes, based upon Canadian Diabetes Association guidelines. Further clinical and laboratory evaluation to verify presence of DPN occurred. Age of DM diagnosis, duration of DPN, and use of anti-diabetes treatments and other medications were documented. Any history of other systemic illness, toxic and medication exposure, alcohol use, and familial neuropathy was determined. All recruited subjects performed laboratory testing at study initiation and completion to identify other peripheral neuropathy causes [13]. Hemoglobin A1C, electrocardiography and hepatic and renal function blood work were performed at the beginning and end of each intervention period.

\section{Subject assessment}

During baseline week, assessments and questionnaires were completed. Daily pain severity and associated clinical reporting diaries were initiated at baseline week, at the end of first intervention, during the second week of the washout phase, and at the end of second intervention. During each of the six week intervention periods, daily pain diaries were completed. Adverse events were recorded during each visit.

A timeline for all visits is presented in Additional file 1: Figure S1. At screening and final visits, a complete standardized neurological examination and full physical examination was carried out [13]. Each patient was scored using the Utah Early Neuropathy Scale (UENS) determining DPN severity [14]. After clinical scales were completed, candidates were verified to have DPN if the UENS score was $\geq 6$. UENS scores of $<6$ led to exclusion due to uncertainty regarding DPN presence. Verification for PDPN presence was based upon the question "Do you have pain on a daily or near daily basis?" If affirmative, candidates then completed the Douleur Neuropathique (DN) 4 questionnaire $(\mathrm{DN} 4 \mathrm{Q})$, categorizing pain as $\mathrm{NeP}$ or non-NeP [15]. Candidates scoring $\geq 4$ on the DN4Q were categorized as PDPN, otherwise exclusion occurred. Pain duration must have been $\geq 3$ months with pain severity at least $40 / 100 \mathrm{~mm}$ on the visual analog scale (VAS) (Short-Form-McGill Pain Questionnaire) [16].

\section{Allocation and criteria}

A randomization table assigned subjects to the first cross-over intervention phase. Randomization concealment occurred for subjects, the clinical coordinator, and the study physician. Scheduled study portions were as follows: 1) Pre-screening to allow wash-out of any prohibited medications (see below); 2) a one week screening period to ensure subject eligibility into the study; 3 ) a six week randomized active treatment period (Intervention 1); 4) a two week washout period with weaning of pregabalin/placebo; 5) a six week randomized active treatment period (Intervention 2); and 6) a one week follow-up period with weaning of placebo/ pregabalin (Figure 1). Telephone visits took place one week into each intervention period, and one week after the washout period to assess tolerability and adverse effects.

Inclusion and exclusion criteria are provided in Table 1. This was an adjuvant study - other pharmacotherapies for pain relief excluding gabapentinoids were permitted 


\section{Investigating the role of neuropathic pain relief for decreasing gait variability in}

diabetes mellitus patients with neuropathic pain

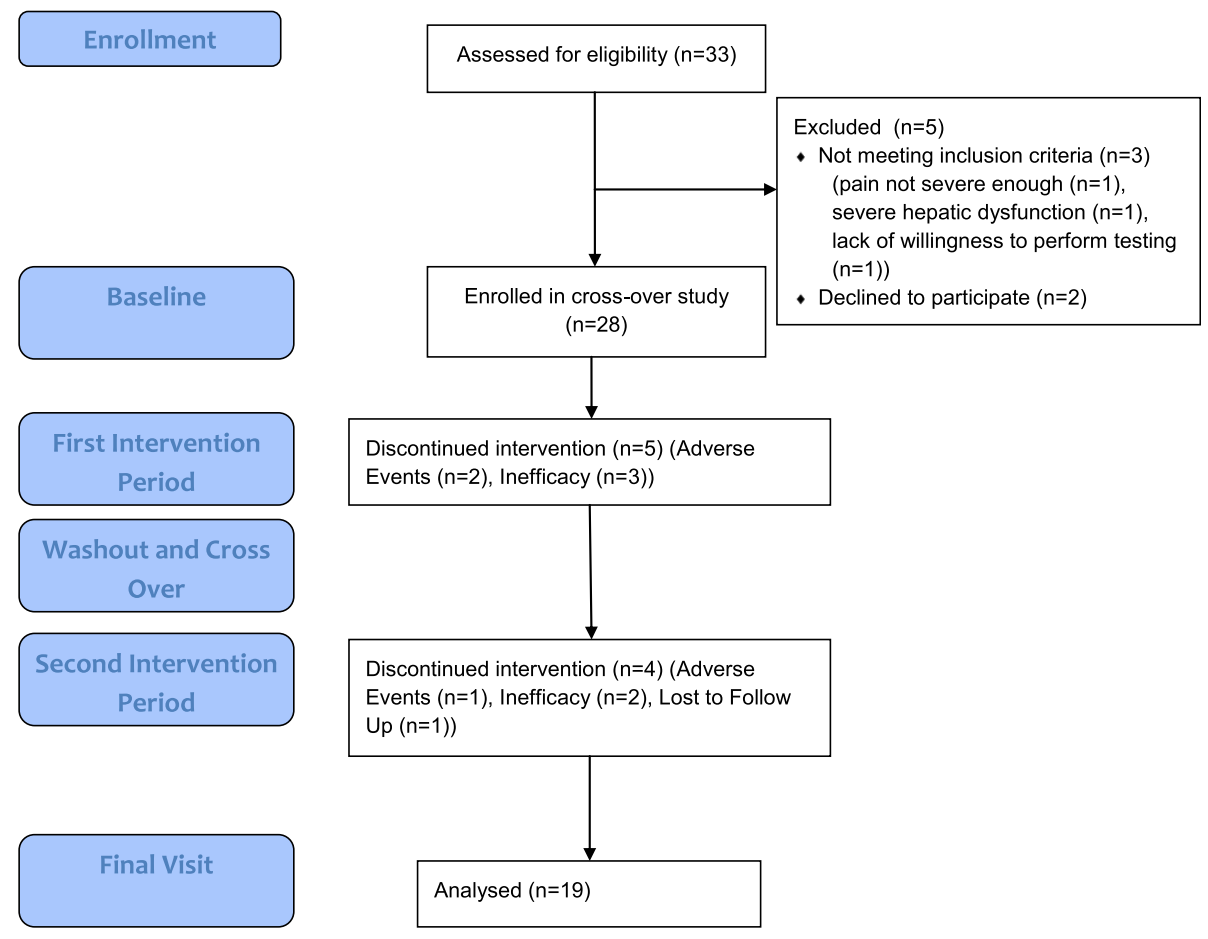

Figure $1 \mathrm{~A}$ flowchart of subjects throughout the study using the CONSORT guidelines.

if stable from 1 month prior to study initiation to end of study. Prior use of pregabalin was permitted unless inefficacy or intolerability arose after appropriate dosing.

\section{Pharmacological interventions}

For pregabalin interventions, subjects were commenced on $75 \mathrm{mg}$ po bid, with scheduled times to increase their dose once weekly after initiation to $150 \mathrm{mg}$ po bid and then $300 \mathrm{mg}$ po bid (maximum dose) if tolerated and agreed upon. A decrease in dose was permitted on one occasion to no less than $75 \mathrm{mg}$ po bid. Tablets supplied were of $75 \mathrm{mg}$ size. Placebo tablets were identical in appearance, size, color, taste, and smell and dosing.

\section{Outcome measures}

GaitMeter $^{\text {TM }}$ analysis was performed prior to and after completion of 6 weeks for each pharmacological intervention. As described [9], GaitMeter ${ }^{\mathrm{Ts}}$ is a small, wireless, portable, inertia-sensing motion analysis system using miniature accelerometers and gyroscopes attached to the anterior mid shin regions permitting various mobility tasks in a natural environment while data is continuously stored on a personal handheld computer. Subjects walked at normal pace for 50 metres using one 90 degree turn without rest time allowed. Data collection occurred between a few seconds before gait commencement until a few seconds after conclusion. Step length was measured individually for each leg and averaged in all cases using tibial acceleration, as analyzed using LabView $^{\text {DM }}$ (National Instruments, Austin, TX) software. Although obtained, angular velocity measures were not studied due to the linear nature of the task studied with GaitMeter $^{\mathrm{Tx}}$. The first and last steps were not analyzed, as these are not typically a full step and would contribute to variability incorrectly if included. Step velocity was calculated using the distance and time of each individual leg's step as measured by GaitMeter ${ }^{\mathrm{Tm}}$. The degree of statistical variability in each of gait step distance and velocity was used to calculate measures of gait variability, defined as the extent to which the individual data diverged from the average value using variance. Although GaitMeter ${ }^{\mathrm{rm}}$ has been studied previously and found reliable, this device has not been validated against an established device or procedure for evaluation of gait in this manner. Secondary outcome measures included assessment of the mean difference in the average daily pain score, averaged from the final seven entries in the daily pain diary during the last week of the intervention period. Comparison was performed with average scores obtained in either the baseline week or in the last week of the washout period prior to 
Table 1 Inclusion and exclusion criteria for study entry are listed

\begin{tabular}{|c|c|}
\hline \multirow[t]{4}{*}{ Inclusion criteria } & 1) Subjects aged $\geq 18$ years \\
\hline & $\begin{array}{l}\text { 2) Females of childbearing potential must have a negative urine pregnancy test performed, and all subjects were required to be } \\
\text { practicing an effective form of contraception as required }\end{array}$ \\
\hline & 4) Ability to complete pain diary and quality of life questionnaires and to perform GaitMeter ${ }^{\mathrm{TM}}$ and associated testing; and \\
\hline & 5) The subject must be willing and able to comply with scheduled study procedures. \\
\hline & 2) Clinically significant or unstable medical or psychological conditions \\
\hline & $\begin{array}{l}\text { 3) Any history of malignancy, except either where there has been no ongoing treatment for at least } 6 \text { months or for a basal cell } \\
\text { carcinoma }\end{array}$ \\
\hline & $\begin{array}{l}\text { 4) A history of seizures, clinically significant cardiac arrhythmias, postural hypotension, uncontrolled hypertension, severe } \\
\text { cardiovascular disease, severe hepatic impairment (evidence from medical history), renal failure or pulmonary disease }\end{array}$ \\
\hline & $\begin{array}{l}\text { 6) Hepatic dysfunction at screening (aspartate transaminase [AST] or alanine transaminase [ALT] greater than twice the upper limit } \\
\text { of normal, or a total bilirubin greater than the upper limit of normal }\end{array}$ \\
\hline & 7) Estimated creatinine clearance of $<60 \mathrm{~mL} / \mathrm{min}$ based on the Cockcroft and Gault equation \\
\hline & 8) A positive urine drug screen \\
\hline & 9) A lack of willingness to discontinue and wash-out use of prohibited medications or treatments \\
\hline & 10) Inability to walk a total of 50 metres without any pauses or use of a cane, walker, or motorized device \\
\hline & $\begin{array}{l}\text { 11) Identification of the presence of another potential cause for peripheral neuropathy, presence of impaired glucose tolerance } \\
\text { only or juvenile onset of diabetes with requirement for insulin at time of diagnosis (i.e. possible type } 1 \text { diabetes) }\end{array}$ \\
\hline & boratory and physiological testing. \\
\hline
\end{tabular}

each intervention period. Pain severity was rated daily using an 11-point numerical rating scale (NRS) from $0=$ no pain to $10=$ worst possible pain using an average score for the preceding 24 hours.

Secondary physiological outcome measures were performed at baseline week, at the last week of the washout period, and at the end of each intervention period. A Physiological Profile Approach (PPA) [17] was performed - this tests high and low contrast visual acuity, visual field dependence, peripheral sensation (tactile, vibratory, and proprioceptive sensation), muscle force using dynamometry, reaction time, and balance using standard clinical measures and complimentary measures. Corrected visual acuity testing was performed using a standardized Snellen eye chart. Proprioceptive testing was performed at the knee as described [17], with scoring in degrees of disparity between levels of the great toes. Contrast sensitivity was performed using the Vistech chart to determine orientation of gratings provided in rows of circles differing in contrast [18], scored as logarithmic values of inverses. Blood pressure measurements for lying, sitting, and standing were performed using one minute latencies. Mobile handheld dynamometry using a Chatillon MSE100 dynamometer (Digital Measurement Metrology, Brampton, ON) for ankle dorsiflexion and plantar flexion [19] was performed. Reaction times of the dominant foot using a visual stimulus and recording of latency for the foot tapping on a measurement device. Balance testing used four scenarios to measure postural sway [17] - standing on floor with eyes open or closed, and standing on $15 \mathrm{~cm}$ thick foam with eyes open or closed, each for 30 seconds duration. Sway was calculated using a rod fixed to the posterior aspect of a belt while on the opposite end, a computer tablet pencil would scroll on touch-sensitive computer screen to measure percentages of movement based upon the computer screen area. Additional testing for determination of fall risk included the Timed Get-Up-and-Go Test (TUG) [20] and the Tinetti Assessment Tool (TAT) [21]. All subjects also completed the Modified Falls Efficacy Scale (MEFS) [22] and the Elderly Falls Screening Test (EFST) [23] at $0,2,6$ and 8 weeks into each intervention.

All adverse events, both spontaneously reported and observed, were coded during each clinic or telephone visit using Medical Dictionary for Regulatory Activities (MedDRA) terminology. Vital signs and body weight were measured at clinic visits. Electrocardiograms were performed at screening and final visits.

\section{Analysis}

The primary endpoint was defined as a measurable difference in the absolute gait variability using GaitMeter after 6 weeks of each intervention. Based upon the primary outcome measure, assuming a 2-sided alpha of 0.05 with a standard deviation of 0.02 (SD 0.02), a 
sample size was calculated for 25 patient subjects providing $90 \%$ power to avoid a type 1 error of $<0.01$ to ensure reliability. We anticipated a $10 \%$ drop out rate, and therefore 28 subjects were to be recruited.

The primary outcome measure analyzed was intrasubject variability in gait for step length and velocity considering all steps made by both limbs except for first and last steps of the 50 metre walk. Intra-subject variability calculations averages were obtained for all subjects during each intervention. Shapiro-Wilk testing was performed to ensure normal distributions prior to comparisons of variance for variables of interest. If normality was determined, then Bartlett-Box testing was performed to assess for homogeneity of variance between cohorts, using the null hypothesis that both interventions were associated with the same degree of variance for gait variables of interest. In the case of non-normal distributions for variance, general multivariate analysis of covariance (MANCOVA) testing using intra-subject variability in gait step length and step velocity as dependent measures with intervention was used as an independent measure. MANCOVA testing can be used when there are multiple dependent variables, yet significant differences between group means are sought. Each of the above tests were previously used in our work preliminary to this study [9]. Interlimb differences for each subject were compared for step length and velocity. Post-hoc linear regression was performed for determining relationships of gait variability with measures of step length, step velocity, and pain severity, using pain severity as a dependent variable, and step velocity and step length as independent or dependent variables depending upon the assessment performed. Post-hoc comparisons in the change in variability between baseline and endpoint timepoints have also been performed as a comparison between the two interventions administered. Absolute changes in variables were examined for significant differences for all variables examined at different time points. Separate ANOVA testing with calculation of $\mathrm{F}$ values was performed using Bonferroni corrections for the remaining secondary and tertiary outcome measures. $\alpha$ was set to be 0.05 . Only data received from patients completing the entire study was analyzed. Analysis occurred using Microsoft Excel 2007 (Office 2007, Microsoft Corp, Washington State) or SPSS 14.0 (IBM, Armonk, NY). An intention-to-treat analysis could not be performed due to the primary outcome measure being dependent upon the gait assessments occurring at both the first and last week for each intervention.

\section{Results}

\section{Participants}

We recruited 28 subjects after assessing 33 potential candidates (Figure 1). A total of 19 subjects completed the entire study; discontinuations occurred in both intervention periods due to inefficacy of pain relief and intolerability, with one patient lost to follow-up for uncertain reasons (Table 2). There was no significant difference in the numbers of other pain medications used between baseline and endpoint times, or between the two interventions performed for either baseline and endpoint timepoints.

Gaitmeter $^{\mathrm{TM}}$ assessment revealed no significant differences in durations of time required to walk the entire 50 metres (Table 3) (ANOVA, $\mathrm{p}=\mathrm{NS}, \mathrm{F}=0.3-0.7$ ). Average actual step length and step velocity measures were unchanged between timepoints and interventions (ANOVAs, $\mathrm{p}=\mathrm{NS}, \mathrm{F}=0.5-0.9$ ). The baseline degrees of both step length and velocity variance varied between pregabalin and placebo intervention periods, with the placebo intervention demonstrating greater variability for both measures (Bartlett-Box tests, $\chi^{2}=4.49-5.27, \mathrm{p}=0.022-0.034$ ). In contrast to our primary hypothesis, the degree of variability in both step length and step velocity significantly increased for subjects receiving pregabalin for comparison of baseline and final visits (Bartlett-Box tests, $\chi^{2}=5.13$ 9.27, $\mathrm{p}<0.025)$. Although step length variability did not change during the placebo intervention (Bartlett-Box test, $\left.\chi^{2}=1.23, \mathrm{p}=\mathrm{NS}\right)$, step velocity variability decreased during the placebo intervention (Bartlett-Box test, $\chi^{2}=$ $17.07, \mathrm{p}<0.001)$. None of the cohorts had greater variability with one leg as compared to the other leg (BartlettBox tests, $\chi^{2}=0.62-1.11, \mathrm{p}=\mathrm{NS}$ ). Despite differences in variability, there were no significant differences between interventions with respect to step length or step velocity (MANOVAs, $\mathrm{p}=\mathrm{NS}, \mathrm{F}=0.7-1.0$ ). However, there was a difference in the change in variability from baseline to endpoint measurements for each of step length variability (Bartlett-Box test, $\chi^{2}=6.22, \mathrm{p}=0.01$ ) and step velocity variability (Bartlett-Box test, $\chi^{2}=9.57, \mathrm{p}=0.001$ ). Finally, there were no significant differences for individuals between the right and left legs for either of step length or step velocity variability (ANOVAs, $\mathrm{p}=\mathrm{NS}, \mathrm{F}=0.2-0.3$ ).

Subjects during the pregabalin intervention period received non-significant pain relief overall when compared to placebo (Additional file 2: Figure S2). There were no significant differences in pain scores at any timepoint based upon either change from baseline or for the cohort's actual pain scores for each timepoint (multiple ANOVAs, $\mathrm{p}=\mathrm{NS}, \mathrm{F}=0.6-3.7$ ). Scoring for disruption of sleep due to pain had a similar nonsignificant representation (Additional file 3: Figure S3). Although the average score on the MEFS improved initially with pregabalin intervention, overall scoring representation was not significantly different between interventions (Additional file 4: Figure S4).

There were no significant differences for each of high and low contrast visual acuity, proprioceptive thresholds, 
Table 2 Subject characteristics

\begin{tabular}{|c|c|c|c|}
\hline & All study subjects $(n=28)$ & \multicolumn{2}{|c|}{ Subjects completing study $(n=19)$} \\
\hline Age & $64.6 \pm 10.4$ years & \multicolumn{2}{|l|}{$65.7 \pm 10.8$ years } \\
\hline Gender (males) & 17/28 (61\%) & \multicolumn{2}{|l|}{$16 / 19(84 \%)$} \\
\hline Duration of Diabetes Mellitus & $7.2 \pm 3.9$ years & \multicolumn{2}{|l|}{$7.5 \pm 4.1$ years } \\
\hline Duration of Neuropathic Symptoms & $5.7 \pm 4.1$ years & \multicolumn{2}{|l|}{$6.0 \pm 4.3$ years } \\
\hline \multicolumn{4}{|l|}{ Concomitant Diabetic } \\
\hline Complications Present (other than neuropathy) & $5 / 28(18 \%)$ & \multicolumn{2}{|l|}{$3 / 19(16 \%)$} \\
\hline Other Neuropathic Pain Medications Used & $1.2 \pm 1.1$ & \multicolumn{2}{|l|}{$1.4 \pm 1.2$} \\
\hline Weight (kg) & $96.3 \pm 26.8$ & \multicolumn{2}{|l|}{$94.6 \pm 25.9$} \\
\hline Height (cm) & $170.9 \pm 7.6$ & \multicolumn{2}{|l|}{$169.7 \pm 7.8$} \\
\hline Sitting Systolic Blood Pressure (mm Hg) & $141 \pm 18$ & \multicolumn{2}{|l|}{$139 \pm 18$} \\
\hline Sitting Heart Rate (/min) & $72 \pm 10$ & \multicolumn{2}{|l|}{$74 \pm 12$} \\
\hline Standing Systolic Blood Pressure (mm Hg) & $140 \pm 19$ & \multicolumn{2}{|l|}{$136 \pm 20$} \\
\hline Standing Heart Rate (/min) & $73 \pm 12$ & \multicolumn{2}{|l|}{$73 \pm 13$} \\
\hline Utah Early Neuropathy & $16.3 \pm 9.6$ & \multicolumn{2}{|l|}{$16.2 \pm 6.5$} \\
\hline \multicolumn{4}{|l|}{ Score } \\
\hline \multirow[t]{2}{*}{ Douleur Neuropathique 4 Score } & $7.6 \pm 1.0$ & \multicolumn{2}{|l|}{$6.9 \pm 0.9$} \\
\hline & & Block 1 Pregabalin Intervention & Block 1 Placebo Intervention \\
\hline \multicolumn{2}{|l|}{ Baseline Visual Analog Pain Score } & $6.1 \pm 1.6$ & $7.1 \pm 1.3$ \\
\hline \multicolumn{2}{|l|}{ Average Pregabalin } & \multirow[t]{2}{*}{$205.8 \pm 28.9$} & \multirow[t]{2}{*}{$188.1 \pm 39.8$} \\
\hline \multicolumn{2}{|l|}{ Dosage Achieved (mg) } & & \\
\hline Other Neuropathic Pain Medications Used & & $1.4 \pm 1.2$ & $1.4 \pm 1.2$ \\
\hline
\end{tabular}

Values shown are means \pm standard deviations.

and dynamometry-measured forces for leg muscles (ankle dorsiflexion and ankle plantar flexion) (Additional file 5: Table S1) or with balance testing, reaction times, TUG times, TAT scores, and EFST scores (Additional file 4: Figure S4). There were no changes in corrected visual acuity during the course of the study for any subject.

We used multiple linear regression analyses to identify positive associations between step length and step velocity $\left(\mathrm{F}=12.5, \mathrm{R}^{2}=0.38, \mathrm{p}<0.0125\right)$, and for a negative relationship between step length and pain severity ( $\mathrm{F}=$ $\left.10.9, \mathrm{R}^{2}=0.33, \mathrm{p}<0.0125\right)$, but not for step velocity and pain severity $\left(\mathrm{F}=7.1, \mathrm{R}^{2}=0.24, \mathrm{p}=\mathrm{NS}\right)$.

\section{Discussion}

Not as hypothesized, subjects with PDPN receiving pregabalin treatment had increasing variance for both step length and step velocity. Surprisingly, subjects receiving placebo had the unanticipated result of a decrease in step velocity variance (but not for step length variance). These differences occurred without other noted differences, and we interpret these results as related to the interventions themselves. Also, it is important to note that the clinical importance of gait variability is unclear, and is not associated with any particular clinical and obvious outcome. Our results suggest that management of pain relief may

Table 3 Results of gait testing

\begin{tabular}{|c|c|c|c|c|}
\hline Characteristic & $\begin{array}{l}\text { Pregabalin intervention - } \\
\text { baseline }(n=19)\end{array}$ & $\begin{array}{l}\text { Pregabalin intervention - } \\
\text { final }(n=19)\end{array}$ & $\begin{array}{l}\text { Placebo intervention - } \\
\text { baseline }(n=19)\end{array}$ & $\begin{array}{l}\text { Placebo intervention - } \\
\text { final }(n=19)\end{array}$ \\
\hline Cadence (Steps/Minute) & $137.0 \pm 24.9$ & $126.5 \pm 24.2$ & $137.4 \pm 21.9$ & $140.6 \pm 22.9$ \\
\hline $\begin{array}{l}\text { Duration of Time to Walk } 50 \text { Metres } \\
\text { (seconds) }\end{array}$ & $39.8 \pm 0.8$ & $40.2 \pm 1.1$ & $39.7 \pm 0.7$ & $39.5 \pm 0.9$ \\
\hline Average Step Length (metres) & $0.55 \pm 0.11$ & $0.59 \pm 0.13$ & $0.55 \pm 0.15$ & $0.54 \pm 0.15$ \\
\hline Average Step Duration (seconds) & $0.44 \pm 0.08$ & $0.47 \pm 0.09$ & $0.44 \pm 0.07$ & $0.43 \pm 0.07$ \\
\hline Step Length Variance & $0.060 \pm 0.002$ & $0.087 \pm 0.010^{*}$ & $0.098 \pm 0.005$ & $0.100 \pm 0.003$ \\
\hline Step Velocity Variance & $0.043 \pm 0.001$ & $0.066 \pm 0.003^{*}$ & $0.058 \pm 0.003^{\psi}$ & $0.038 \pm 0.000^{k}$ \\
\hline
\end{tabular}

Values shown are means \pm standard deviations. MANOVA testing was used for step length/velocity variance comparisons. ANOVA testing was used for other measures shown. ${ }^{*} p<0.025$ for comparison of baseline and final visit testing in the pregabalin intervention period. ${ }^{\Psi} p<0.025$ for comparison of baseline visit testing for the pregabalin and placebo intervention periods. ${ }^{\mathrm{k}} \mathrm{p}<0.025$ for comparison of baseline and final visit testing in the placebo intervention period. 
not improve gait variability in PDPN subjects, although the pregabalin intervention in our trial did not provide significant pain relief limiting full interpretation of this trial's results.

The presence of PDPN contributed to greater gait variability in our prior work [9], with potential contribution to postural instability and even falls. We hypothesized that pain itself contributed to this variability and development of an overly cautious antalgic gait with smaller steps. However, the addition of pregabalin may negatively impact gait function. Rats receiving extreme pregabalin doses have a dose-dependent impairment in rotarod performance [24]. Doses administered in our study were about 1000 fold less by weight, but the sensitivity of GaitMeter ${ }^{\mathrm{Ts}}$ may be sufficient to identify small changes in human gait. Rats receiving phenytoin or carbamazepine are subject to much more profound ataxia [24]. Although ataxia was not reported as an adverse effect, it has been reported in addition to vertigo, nystagmus, incoordination and balance disorders as due to pregabalin [25], but with less magnitude than phenytoin and carbamazepine [26]. As such, it is possible that pregabalin's adverse effects may have contributed to our findings.

There was a non-significant improvement with pain relief using pregabalin as a large confounder in this trial; if greater pain relief had occurred, it is possible that improved gait variability may have been observed. However, the discovery of placebo intervention's association with reduced gait variability was unanticipated. In studies examining gait parameters in patients with knee osteoarthritis, placebo did not impact upon outcome measures [27]. The degree of placebo response is highly variable [28] and subject to particular personality types [29]. Other studies, however, have identified placeboassociated improvements in gait and balance [30]. Furthermore, subjects with low back pain are subject to reduced variability of gait [12], so perhaps the PDPN population has other distinct features leading to gait variability rather then the chronic pain itself.

Factors increasing gait dysfunction [31] include stride time variability, poor functional status or physiological capacity. Exercise programs may benefit instability [31]. Otherwise, management of gait dysfunction largely depends upon the disease process. Management of Parkinsonism and cardiovascular conditions, for example, improves gait dysfunction. Improvement in postural hypotension or discontinuation of medications causing ataxia or sedation can also improve gait. Fall prevention techniques are also of value, including gait assistance devices.

DPN patients have other contributing factors for falling [6] beyond NeP. Proprioceptive and cutaneous sensory loss also contributes. An antalgic gait in PDPN patients also contributes to instability [11]. Antalgic gait contributes to slow walking along with increased step frequency, reduced step length, less lateral sway in center-of-mass, and reduced amounts of ankle plantar flexion and hip extension movements, resulting in greater gait variability [9]. However, treatment of PDPNassociated $\mathrm{NeP}$ failed to reduce gait variability and, in fact, increased gait variability. This suggests that NeP management is unlikely to reduce risks of falling if of insignificant amount, but it remains unclear if significant pain relief may impact upon gait dysfunction.

Our findings should not dismay other research teams from identification of other potential risk factors for falling in DM. There are limitations to our results. The largest limitation was the absence of successful pain relief with pregabalin, either based upon change from baseline pain severity or as an absolute difference. This insufficient pain relief may have been due to a number of possible factors including a type II error, insufficient dosing, insufficient duration of treatment, or more refractory pain in our patient population. Also, the design of the study using both baseline and endpoint gait measures limited us from performance of an intention-totreat analysis. The presence of greater baseline gait variability in the placebo intervention portions was unanticipated, and suggests that randomization may have been better performed using a stratified approach after baseline gait measures were performed. Calculation of foot contact moments and other potentially useful measures could not be performed using GaitMeter ${ }^{\mathrm{TM}}$. We did not formally assess cognitive function or vestibular function. Our tertiary care patients enrolled may not be representative of primary care populations. Although sample size calculations were met, discontinutations were more frequent than expected, dampening our results. Training effects over the four GaitMeter ${ }^{\mathrm{Tm}}$ sessions performed may have influenced the results of the second intervention, and incomplete washouts may have occurred. GaitMeter $^{\mathrm{rm}}$ has been shown reliable in this patient population based upon our prior work, but has not received validation with comparison against established gait analysis devices or procedures; as such, this invalidation may have contributed to the unanticipated results observed. Although other pain relief medications were stable, they may have impacted upon results. There were large degrees of variance in many examined measures, particularly with the baseline timepoint gait measures in the placebo intervention group. Finally, the lack of significant pain relief with pregabalin makes interpretation of these results difficult.

\section{Conclusion}

Our results suggest that although PDPN impacts upon gait stability [9], its potential relief using pharmacotherapy may not improve gait dysfunction. Future studies with larger sample sizes and other forms of management 
for $\mathrm{NeP}$ should be considered. Although our hypothesis was not met, management of PDPN is clearly associated with other significant benefits related to pain relief, improved functionality and quality of life. We suggest that further kinesiological assessments of patients with PDPN are required to determine the reasons behind gait variability and its potential future management.

\section{Additional files}

Additional file 1: Figure S1. A timeline for all visits in this trial is presented. In person visits are demonstrated by $V_{1}, V_{2}$, etc. while telephone visits are not shown. During screening, informed consent is obtained. History and physical examination, along with electrocardiography, blood work, recording of number of falls, urine pregnancy testing and DN4 questionnaires are performed on visits marked with a plus sign (+). After the run in phase and at the time of randomization to either of pregabalin or placebo, gait and physiological assessments, along with performances of all other questionnaires are performed for visits shown with an asterisk (*). During the time of cross over to the other intervention, a 2 week washout period occurs during weeks $7-8$. The final visit occurs two weeks after the completion of the second intervention (or with study dropout) as indicated.

Additional file 2: Figure S2. The impact of interventions upon VAS pain severity levels. Over the time of the second intervention, the cohort receiving placebo followed by pregabalin had significantly better pain relief during the second intervention as compared to the cohort receiving pregabalin, then placebo (informal post-hoc analysis, ANOVA, $p<0.05)(\mathbf{A})$. However, when subjects are grouped according to intervention received, there was a non-significant pain relief identified with the pregabalin intervention (ANOVA, $\mathrm{p}=\mathrm{NS}$ ) (B). Values shown are means \pm standard error

Additional file 3: Figure S3. The impact of interventions upon VAS sleep disturbance severity levels. As with the pain severity measure, the cohort receiving placebo followed by pregabalin had significant improvement in sleep disturbance during the second intervention as compared to the cohort receiving pregabalin followed by placebo (informal post-hoc analysis, ANOVA, p < 0.05) (A). However, when grouped based upon intervention received, there was a non-significant impact upon sleep disturbance during the pregabalin intervention (ANOVA, $p=N S$ ) (B). Values shown are means \pm standard error.

Additional file 4: Figure S4. Scores on the Modified Efficacy Falls Score were not significantly different between interventions (ANOVA, $p=N S$ ) (A). Likewise, performance on balance testing did not change with either intervention and was similar for all time points measured (multiple ANOVAs, $p=N S$ ) (B). Reaction times were unchanged for any of the studied timepoints also (ANOVA, $p=N S$ ) (C). Other assessments of mobility, including the Tinetti Up and Go time (D), Tinnetti Assessment Tool (E), and the Elderly Fall Screening Test score (F) were also unchanged between interventions (multiple ANOVAs, $p=N S$ ).

Additional file 5: Table S1. Results of ancillary testing.

\section{Competing interests}

Cory Toth has previously received honoraria from Pfizer Canada for medical education seminars performed, as well as previously receiving both clinical and preclinical research funding for studies related to pregabalin. Cory Toth has also received honoraria for medical education seminars from Lilly Canada and Johnson \& Johnson over the past 5 years.

\section{Authors' contributions}

HR and SK performed data entry and data sorting, along with portions of data analysis and both reviewed the manuscript. CC organized the clinical interactions, gait assessments, performed and the ancillary laboratory and physiological assessments, and reviewed the manuscript. CT developed the protocol, performed all clinical assessments, provided overview for the data and the study performance, and wrote the first draft of the manuscript. All authors read and approved the final manuscript.

\section{Acknowledgments}

This study was funded through receipt of a Neuropathic Pain Research Award in 2009 from Pfizer Canada. Pregabalin and placebo within the study were provided by Pfizer Canada. This agency had no role in design, conduct of the study, collection, management, analysis or interpretation of the data, review, or manuscript approval. This work has been previously presented at the Peripheral Nerve Society Annual Meeting, St. Malo, France, June 2013. Mr. Edward Block developed the GaitMeter ${ }^{\mathrm{TM}}$, performed gait analysis and assisted with data analysis. Mr. Block holds a patent with respect to GaitMeter ${ }^{\mathrm{TM}}$, but none of the authors associated with this paper have any financial or other interest with respect to GaitMeter ${ }^{\mathrm{TM}}$.

Received: 28 February 2014 Accepted: 25 July 2014 Published: 20 August 2014

\section{References}

1. Malmivaara A, Heliovaara M, Knekt P, Reunanen A, Aromaa A: Risk factors for injurious falls leading to hospitalization or death in a cohort of 19,500 adults. Am J Epidemiol 1993, 138(6):384-394.

2. Yau RK, Strotmeyer ES, Resnick HE, Sellmeyer DE, Feingold KR, Cauley JA, Vittinghoff E, De Rekeneire N, Harris TB, Nevitt MC, Cummings SR, Shorr RI, Schwartz AV: Diabetes and risk of hospitalized fall injury among older adults. Diabetes Care 2013, 36(12):3985-3991.

3. Herzog RI, Sherwin RS: DIABETES can tight glycemic control in diabetes benefit cognition? Nat Rev Neurol 2012, 8(3):124-126.

4. Zochodne DW: Diabetic polyneuropathy: an update. Curr Opin Neurol 2008, 21(5):527-533.

5. Biessels GJ, Deary IJ, Ryan CM: Cognition and diabetes: a lifespan perspective. Lancet Neurol 2008, 7(2):184-190.

6. Cavanagh PR, Derr JA, Ulbrecht JS, Maser RE, Orchard TJ: Problems with gait and posture in neuropathic patients with insulin-dependent diabetes mellitus. Diabet Med 1992, 9(5):469-474.

7. Merskey H, Bogduk N: Classification of Chronic Pain: Descriptions of Chronic Pain Syndromes and Definitions of Pain Terms. Seattle: IASP Press; 1994.

8. Van Acker K, Bouhassira D, De Bacquer D, Weiss S, Matthys K, Raemen H, Mathieu C, Colin IM: Prevalence and impact on quality of life of peripheral neuropathy with or without neuropathic pain in type 1 and type 2 diabetic patients attending hospital outpatients clinics. Diabetes Metab 2009, 35(3):206-213.

9. Lalli P, Chan A, Garven A, Midha N, Chan C, Brady S, Block E, Hu B, Toth C: Increased gait variability in diabetes mellitus patients with neuropathic pain. J Diabetes Complications 2013, 27(3):248-254.

10. Katoulis EC, Ebdon-Parny M, Lanshammar H, Vileikyte L, Kulkarni J, Boulton AJ: Gait abnormalities in diabetic neuropathy. Diabetes Care 1997, 20(12):1904-1907.

11. Barak Y, Wagenaar RC, Holt KG: Gait characteristics of elderly people with a history of falls: a dynamic approach. Phys Ther 2006, 86(11):1501-1510.

12. Seay JF, Van Emmerik RE, Hamill J: Low back pain status affects pelvis-trunk coordination and variability during walking and running. Clinical Biomech 2011, 26(6):572-578.

13. Wile DJ, Toth C: Association of metformin, elevated homocysteine, and methylmalonic acid levels and clinically worsened diabetic peripheral neuropathy. Diabetes Care 2010, 33(1):156-161.

14. Singleton JR, Bixby B, Russell JW, Feldman EL, Peltier A, Goldstein J, Howard J, Smith AG: The Utah early neuropathy scale: a sensitive clinical scale for early sensory predominant neuropathy. J Peripher Nerv Syst 2008, 13(3):218-227

15. Bouhassira D, Attal N, Alchaar H, Boureau F, Brochet B, Bruxelle J, Cunin G, Fermanian J, Ginies P, Grun-Overdyking A, Jafari-Schluep H, Lantéri-Minet M, Laurent B, Mick G, Serrie A, Valade D, Vicaut E: Comparison of pain syndromes associated with nervous or somatic lesions and development of a new neuropathic pain diagnostic questionnaire (DN4). Pain 2005, 114(1-2):29-36.

16. Melzack R: The short-form McGill pain questionnaire. Pain 1987, 30(2):191-197.

17. Lord SR, Menz HB, Tiedemann A: A physiological profile approach to falls risk assessment and prevention. Phys Ther 2003, 83(3):237-252

18. Pesudovs K, Hazel CA, Doran RM, Elliott DB: The usefulness of Vistech and FACT contrast sensitivity charts for cataract and refractive surgery outcomes research. Br J Ophthalmol 2004, 88(1):11-16. 
19. Wadsworth C, Nielsen DH, Corcoran DS, Phillips CE, Sannes TL: Interrater reliability of hand-held dynamometry: effects of rater gender, body weight, and grip strength. J Orthop Sports Phys Ther 1992, 16(2):74-81.

20. Podsiadlo D, Richardson S: The timed "Up \& Go": a test of basic functional mobility for frail elderly persons. J Am Geriatr Soc 1991, 39(2):142-148.

21. Raiche M, Hebert R, Prince F, Corriveau H: Screening older adults at risk of falling with the Tinetti balance scale. Lancet 2000, 356(9234):1001-1002.

22. Hill KD, Schwarz JA, Kalogeropoulos AJ, Gibson SJ: Fear of falling revisited. Arch Phys Med Rehabil 1996, 77(10):1025-1029.

23. Cwikel JG, Fried AV, Biderman A, Galinsky D: Validation of a fall-risk screening test, the Elderly Fall Screening Test (EFST), for communitydwelling elderly. Disabil Rehabil 1998, 20(5):161-167.

24. Vartanian MG, Radulovic LL, Kinsora JJ, Serpa KA, Vergnes M, Bertram E, Taylor CP: Activity profile of pregabalin in rodent models of epilepsy and ataxia. Epilepsy Res 2006, 68(3):189-205.

25. Zaccara G, Gangemi P, Perucca P, Specchio L: The adverse event profile of pregabalin: a systematic review and meta-analysis of randomized controlled trials. Epilepsia 2011, 52(4):826-836.

26. Collaborative Group for Epidemiology of Epilepsy: Adverse reactions to antiepileptic drugs: a multicenter survey of clinical practice. Collaborative group for epidemiology of epilepsy. Epilepsia 1986, 27 (4):323-330.

27. Asay JL, Boyer KA, Andriacchi TP: Repeatability of gait analysis for measuring knee osteoarthritis pain in patients with severe chronic pain. J Orthop Res 2013, 31(7):1007-1012.

28. Turner JA, Deyo RA, Loeser JD, Von Korff M, Fordyce WE: The importance of placebo effects in pain treatment and research. JAMA 1994 271(20):1609-1614.

29. Hauser W, Bartram-Wunn E, Bartram C, Reinecke H, Tolle T: Systematic review: Placebo response in drug trials of fibromyalgia syndrome and painful peripheral diabetic neuropathy-magnitude and patient-related predictors. Pain 2011, 152(8):1709-1717.

30. Janssen M, Stokroos R, Aarts J, van Lummel R, Kingma H: Salient and placebo vibrotactile feedback are equally effective in reducing sway in bilateral vestibular loss patients. Gait Posture 2010, 31(2):213-217.

31. Hausdorff JM, Nelson ME, Kaliton D, Layne JE, Bernstein MJ, Nuernberger A, Singh MA: Etiology and modification of gait instability in older adults: a randomized controlled trial of exercise. J Appl Physiol (1985) 2001, 90(6):2117-2129.

doi:10.1186/1743-0003-11-125

Cite this article as: Karmakar et al:: Investigating the role of neuropathic pain relief in decreasing gait variability in diabetes mellitus patients with neuropathic pain: a randomized, double-blind crossover trial. Journal of NeuroEngineering and Rehabilitation 2014 11:125.

\section{Submit your next manuscript to BioMed Central and take full advantage of:}

- Convenient online submission

- Thorough peer review

- No space constraints or color figure charges

- Immediate publication on acceptance

- Inclusion in PubMed, CAS, Scopus and Google Scholar

- Research which is freely available for redistribution

Submit your manuscript at www.biomedcentral.com/submit
Biomed Central 